\title{
Snake-like Soft Robot Using 2-Chambers Actuator
}

\author{
Hakim Q.A. Abdulrab ${ }^{1}$, Ili Najaa Aimi Mohd Nordin'², Muhammad Rusydi Muhammad Razif ${ }^{2}$, \\ Ahmad Athif Faudzi ${ }^{1,3^{*}}$
}

\author{
${ }^{1}$ Faculty of Electrical Engineering, Universiti Teknologi Malaysia, Johor Bahru, Malaysia \\ ${ }^{2}$ Faculty of Engineering Technology, Universiti Tun Hussein Onn Malaysia, Batu Pahat, Malaysia \\ ${ }^{3}$ Centre for Artificial Intelligence and Robotics, Universiti Teknologi Malaysia, Johor Bahru, Malaysia
}

*Corresponding author: athif@utm.my

\begin{abstract}
Many researchers have been working on snake-like robots due to their flexibility, safety and dexterity. Traditional robots have rigid underlying structures that limit their ability to interact with their environment. In this work, soft robot is developed using three links of the flexible soft actuator connected by rubber joints. The actuators are fabricated using silicon Silastic P-1 where each actuator link consists of two semi-circular chambers and are reinforced with fibers. Fabrication process from CAD design, mold fabrication and validation with simulation and experiment is presented. The fabricated actuators can bend at $27.5^{\circ}$ with maximum pressure of $180 \mathrm{kPa}$.
\end{abstract}

Keywords: flexible actuator, two chambers, three links, finite element analysis, soft actuator.

Article History: received 28 June 2017; accepted 11 April 2018; published 16 April 2018.

(C) 2018 Penerbit UTM Press. All rights reserved.

\section{INTRODUCTION}

Soft manipulators and robots have been explored by many researchers due to their unique characteristics and benefits: flexibility, safety, dexterity and the ability of extension and reduction [1]. Some developing fields of soft robots are in medical robotics [2], bio-inspired legged robots [3], pouch motor [4] and many more.

In the last decade, snake-like robot has become an interesting topic as there were many scholars who started working on this kind of robot. In 1989, Fukuda et al. had developed rubber actuators using Hydrogen Storage Alloy (HSA) for an in-pipe inspection robot, which can move inside of pipelines with only two-inch inner diameter [5]. Many other snake-like robots have been established and have shown extraordinary potential [6]-[9]. The proposed actuators are light in weight and flexible to move in the pipeline. The most interesting thing about snake robots is the dynamics of the robot itself. Ostrowski and Burdick discussed the snake-like robot locomotion theory based on geometric mechanics [10]. Ma developed the dynamics model by applying the Newton- Euler method [11]. Saito et al. decoupled the dynamics of the robot and determined the feedback control architecture [9]. In addition, Andersson approximated a continuous curve with the snake-like robot based on the product-of-exponentials (POE) formula [12]. Liljebäck et al. presented the SnakeFighter concept in the form of a water hydraulic snake robot. The application of the system is focused on fire intervention tasks [7].

Some have studied the dynamics of locomotion while others studied on the dynamics of manipulation [8]. The similarity in the mechanism of locomotor and the manipulator has led to interesting relationships in term of dynamics. This was a motivation which led to defining the dynamic equations for locomotion and manipulation of snake-like robots. These equations were unified by taking the advantage of geometrical formulation. While doing the unifying and comparing process, the unified dynamics offer understanding beyond the separate ones.

Shuichi Wakimoto et al. in his research applied the snake-like locomotion to the robot constructed by a tiny link mechanism and micro DC motors [13]. The structure and the driving mechanism of this locomotion consist of 7 links and uses 6 rotational joints. Sine wave signals are used to drive the rotational joints with small phase differences between touching. Imitating the body of a snake, the structure of a snake-like robot is an articulated mechanism without a fixed base. Target value of joint position is obtained from following equation:

$$
R(i)=A \sin \left\{\omega t-2 \pi \frac{(i-1)}{\lambda}\right\}
$$

where $i$ represents the identity number of the joint that are numbered from the back to the front of the robot, $\omega$ is the angular frequency, A represents the magnitude of bending angle, $\lambda$ is the number of links shaping one wave named wave length and $t$ is time.

Snake-like robot can be redundant manipulator if it is attached to a fixed based on one of its ends and applied in medical robots [14]. To make the snake like robot powerful and can be used in many fields, it is needed to make locomotion and manipulation integrations. Some of the fields where a snake-like robot can be used are searching and rescuing in disasters, inspecting and repairing in industries [8].

This paper will present a snake-like design using three links of 2-chambers FMA design proposed by Suzumori et 
al [15]. Each actuator has 2 degrees of freedom (DOF) with a total of 6 DOF for the robot. Section 2 will present the system design and discuss the fabrication process. Section 3 will further explain the fabrication of the mold and the actuator. Section 4 will discuss the control system with on/off valve for bending experiment of the snake-like manipulator and finally section 5 will summarize the work.

\section{SYSTEM DESIGN}

There are various fabrication methods of soft actuators using McKibben actuator [16], balloon type [4], bellow shape [17], and with different kind of soft materials. Suzumori et al proposed a simple design of soft actuator fabrication using two chambers concept where the pressure in each chamber is being controlled independently via pneumatic flexible tubes [15][18][19]. To resist the deformation in the radial direction, the silicone rubber is reinforced with nylon cords in the circular direction as shown in the figure as in Figure 1. As the pressure is increased in one chamber, the chamber expands in the axial direction and the actuator bends in the opposite direction of the pressurized chamber.

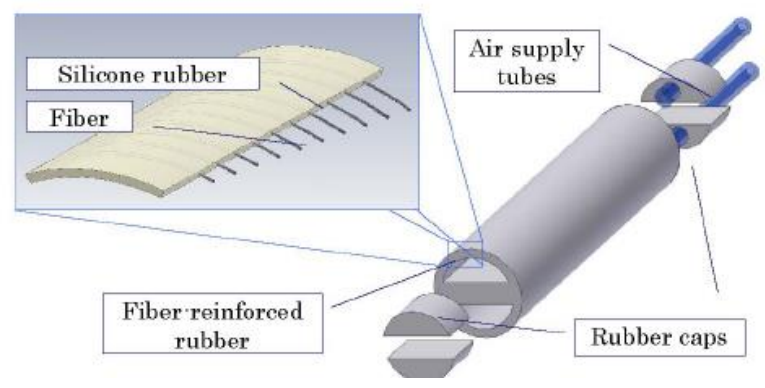

Figure 1. The structure of two-degrees of freedom pneumatic actuator [15]

This actuator could be reshaped so it can be used in many applications. For example, when the actuator is needed to make a robot finger it should suit the shape of grasped object. The actuator extends in the axial direction in case the pressure is applied in both chambers at the same time. As a result, it can be said that the actuator has many abilities such as performing two degrees of freedom, bending and stretching.

The material that is used to build the structure of armtype Flexible Micro Actuators (FMAs) is made of fiberreinforced silicone rubber. Generally, FMAs have three or two internal chambers. By using the control valve, the pressure inside each chamber can be controlled through independent tubes that are connected to the valve. The direction of the fiber-winding differs from type (I) to type (II). For type (I) there is no angle taking when placing the fiber around the actuator. The rubber of this type is reinforced in circular direction therefore the value of the angle $\alpha$ is 0 degree. That is not the case for type (II) where its fiber is reinforced around the actuator with certain range of the angle $\alpha$ from about 5 to 20 degrees. The angle $\alpha$ indicates the direction between fiber orientation and the circular direction.
FMAs can be driven by using analog pressure control servo valves, but in term of system cost and compactness switching valves are more suitable. The simplicity in structure of switching valves makes it possible to be miniaturized sufficiently by using the techniques of microfabrication to be linked directly on the FMAs in the future [20].

Figure 2 shows a pressure control system for an arm type FMA. This system is constructed with three switching valves. Each valve has two positions and three ports. The symbol $\tau$ represents the duty ratio which is the ratio of excitation period, $T p$, to cycle period, $T s$. By adjusting the duty ratio, the pressure $P i$ in the $i^{t h}$ chamber can be easily controlled.

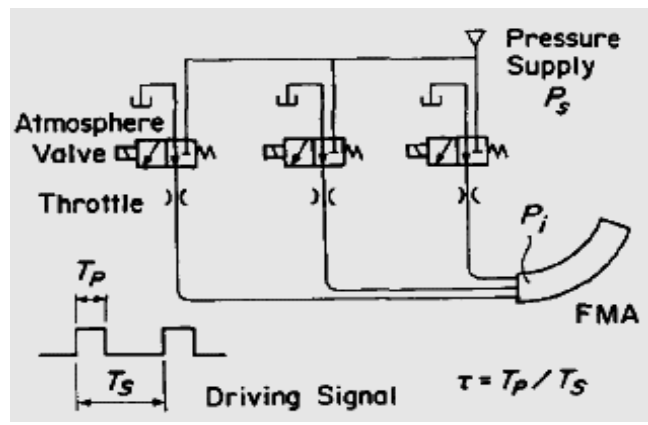

Figure 2. FMA's control system of pressure by pulse width modulation (PWM) valves [20]

Table 1: Characteristics of the one unit of actuator

\begin{tabular}{|l|l|}
\hline Parameter: & Unit \\
\hline Length of actuator & $150 \mathrm{~mm}$ \\
\hline Width of actuator & $18 \mathrm{~mm}$ \\
\hline No. of chambers per actuator & 2 chambers \\
\hline Section of actuator & $254.469 \mathrm{~mm} 2$ \\
\hline Weight of actuator & 50 grams \\
\hline Pressure range & $130-150 \mathrm{kPa}$ \\
\hline
\end{tabular}

The characteristics of the actuators are listed in Table 1 above. These specifications need to be considered for the sake of calculating the bending angle as well as to predict the performance of the actuator when it's fabricated.

\subsection{Step in actuator fabrication}

The actuator fabrication process goes through number of steps. There are six steps to fabricate a rubber actuator. It starts with designing the dimensions of the actuator's inner and outer layers by AutoCAD software. The second step is to fabricate the mold by printing these designs on a polyacetal material using $3 \mathrm{D}$ milling machine. Next step is to mix the pure rubber with a rubber hardener then put the mixture inside the mold. Then eliminate the bubbles from the mixture using vacuum chamber and put the mold with the rubber inside the oven for about 30 minutes. With all these steps being done, the actuator is ready to be used for the planned purpose. The process of fabricating the actuator is summarized in Figure 3. 


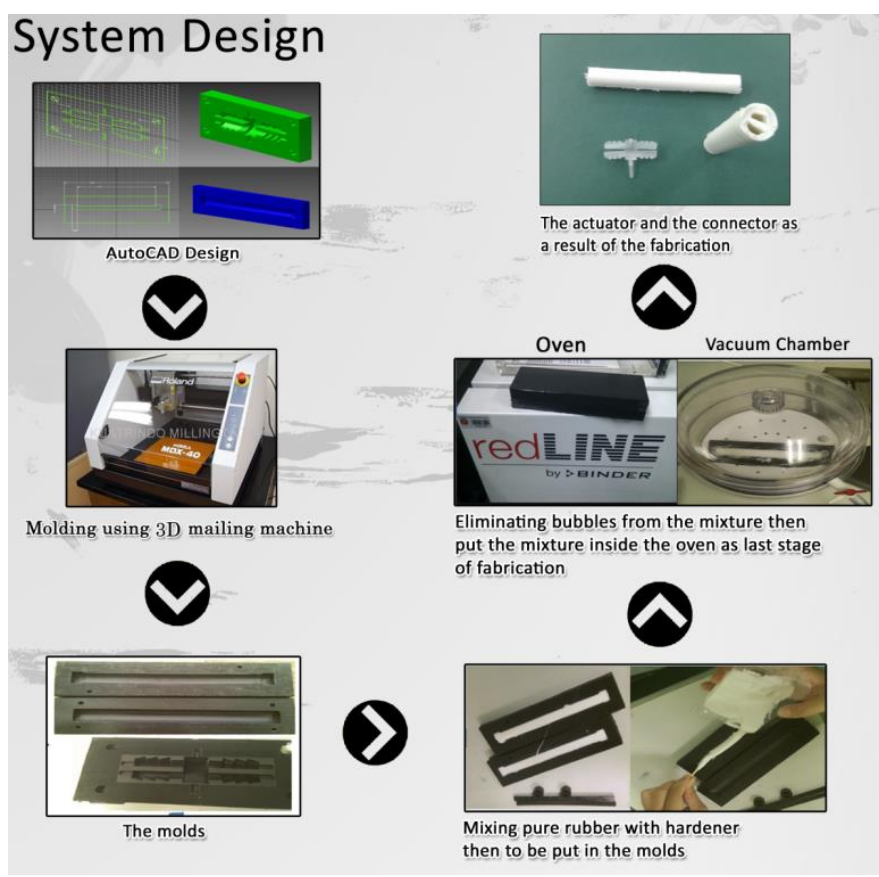

Figure 3. Steps of fabricating the rubber actuators

\subsection{MARC Simulation}

The simulation is done by the finite element methods (FEM) using MARC ${ }^{\text {TM }}$ MENTAT software. The use of the FEM technique helps in predicting the behavior of the model when it comes to the real application. By having good results from the simulation, it can be assured that the real product will behave as expected

MARCTM software is capable to perform nonlinear multi physics analysis. The optimum actuator design can be acknowledged from simulation-based technique. It can save time and money from doing mass trial and error fabrication. The parameters in the FEM simulation need to be specifically set according to the real parameters and conditions in experimental works. The same geometric, material, contact properties and boundary conditions are considered in all models. The inner and outer rubber layers are characterized as natural rubber with following material properties; Hardness (Duro A) 60, ultimate tensile strength 13.6 MPa, Young's modulus 1.2MPa and Poisson's ratio 0.49. It is assumed to be nonlinear homogeneous isotropic hyperelastic material. On the other hand, the fiber layer used between the rubber layers is characterized as pPhenylene-2, 6-Benzobis Oxazole (PBO) and is assumed to be linear, homogenous, isotropic elastic-plastic material, with Young's modulus of $5.8 \mathrm{GPa}$ and Poisson's ratio of 0.3 . The rubber and fiber layers are glued and allowed to deform uniformly.

At first, the simulation was applied to one actuator. A pressure of $150 \mathrm{kPa}$ was applied. The young modulus of the rubber was set to $0.5556 \mathrm{~N} / \mathrm{mm}^{2}$. The inner layer of the actuator is wrapped with Aramid fiber whose young modulus is $3000 \mathrm{~N} / \mathrm{mm}^{2}$ and poison ration of 0.36 . The bending resulted in a major displacement in the $x$ and $z$ directions whereas there was no displacement in the $y$ direction. Figure 4 below shows the displacement.

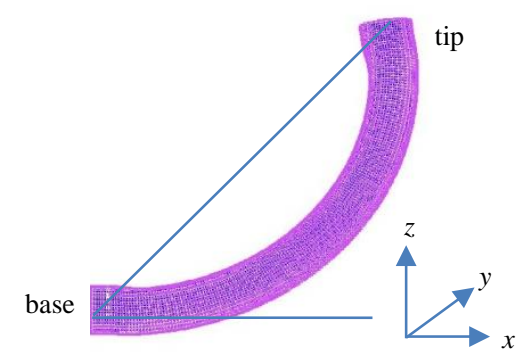

Figure 4. Displacement on the $\mathrm{x}$ and $\mathrm{z}$ axes.

From the simulation data, the displacement of the actuator on the $\mathrm{X}$-axis with respect to the amount of pressure applied is calculated using Equation 2.

$$
y=0.7593 p-2.8697
$$

where $p$ is the supplied pressure.

Similarly, an equation was derived to express the relationship between the displacement on the $\mathrm{z}$-axis and the pressure applied as shown in Equation 3.

$$
z=-0.0036 p^{2}+0.2183 p-2.0547
$$

\section{FABRICATION PROCESS}

To fabricate an actuator, the mold should be designed and fabricated first. Fabricating the mold requires an AutoCAD design.

\subsection{CAD Design}

A single actuator requires eight molds to be fabricated. Six of these molds are needed to fabricate the actuator's inner layer and two for the outer layer. The inner layer is composed of two chambers, two joints and two combiners. On the other hand, the outer layer is composed of two molds. Since the manipulator is composed of three links, connector, base and cover need to be fabricated. The software that is used to design the mold is called Autodesk Inventor.

Four of two chamber molds need to be fabricated. They were designed in a way such each chamber is $160 \mathrm{~mm}$ length and $5 \mathrm{~mm}$ radius. In fact, the length needed is just $150 \mathrm{~mm}$, so the extra $10 \mathrm{~mm}$ will be inserted inside the joints located at both sides to hold these chambers inside the inner layer mold. Figure 5 below shows a screenshot of the chambers with the dimensions being pointed out.

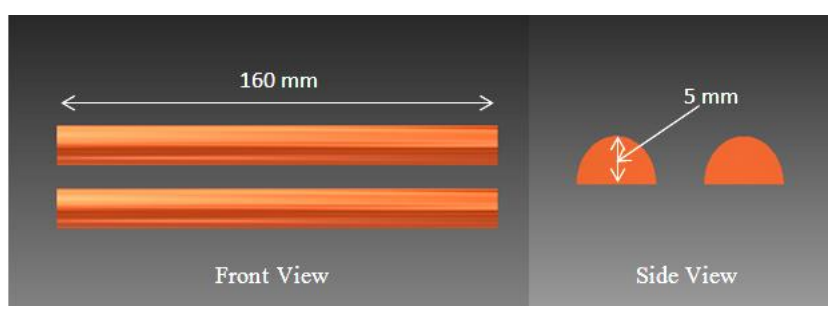

Figure 5. The AutoCAD design of the chamber mold 
To link the three actuators, a bonding joint needs to be fabricated as well. The radius of the joint is slightly bigger than the radius of the chambers to have a flexible insertion at the time of combining the three actuators. As in Figure 6 below, it shows that the joint has an outer diameter of 18 $\mathrm{mm}$ and the inside of it has two semicircles. Each semicircle has a radius of $5.1 \mathrm{~mm}$. The joint has a thickness of $10 \mathrm{~mm}$.

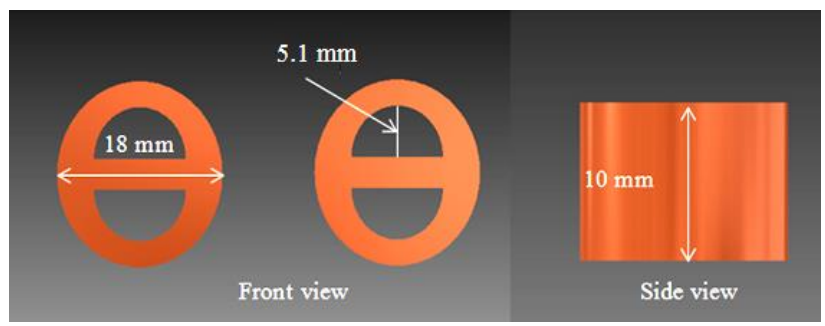

Figure 6. The AutoCAD design of the joint mold

Basically, the design needs two layers molds. The inner layer mold, as in Figure 7, is the one that includes the two chambers and joints. It was designed in a way such the chambers and the joint can be easily inserted. The outer layer length is made up of three original lengths. The total length is $170.2 \mathrm{~mm}$ which is composed of $150 \mathrm{~mm}$ for the chamber length and $10.1 \mathrm{~mm}$ in each end for the joints length. The middle area has a diameter of $14 \mathrm{~mm}$ which is $7 \mathrm{~mm}$ in term of radius. The chamber radius is $5 \mathrm{~mm}$, therefore, the thickness of the inner layer is $2 \mathrm{~mm}$.

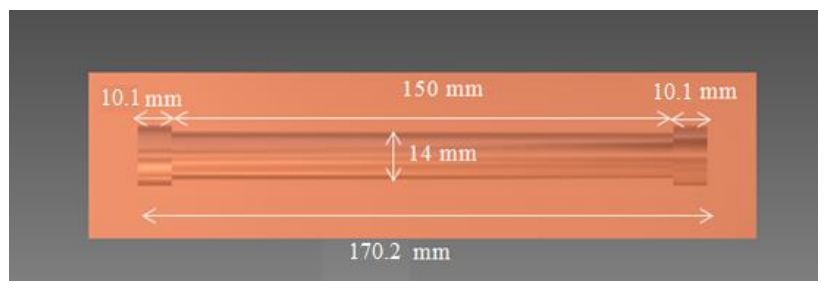

Figure 7. The AutoCAD design of the inner layer mold
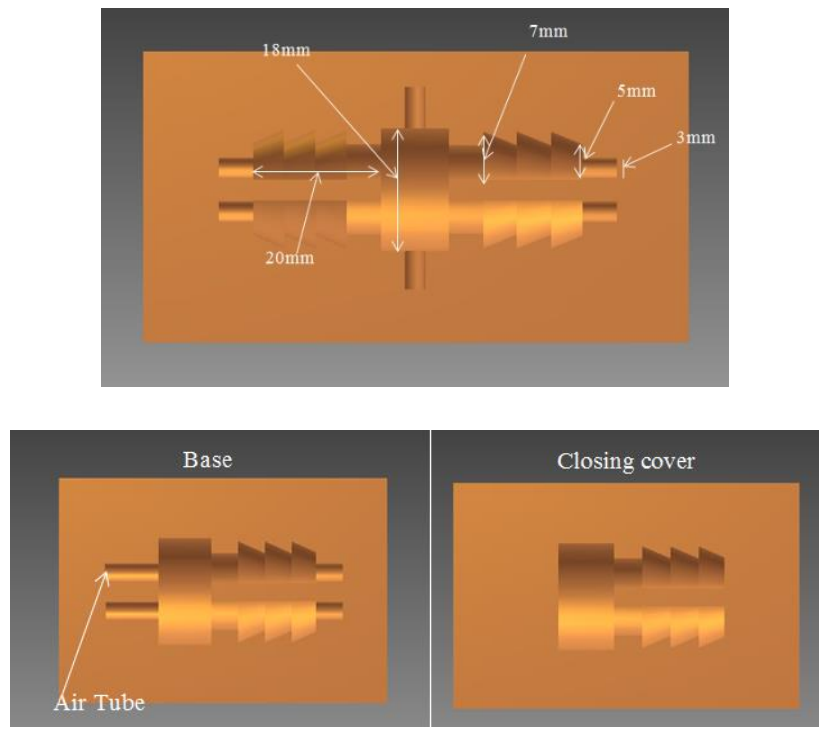

Figure 8 . Design of the connector, base and closing cover
Since the overall design consists of three chambers, a connecter is designed to link these chambers. Figure 8 above shows the design of the connecter along with the base and the end tip.

After fabricating the inner layer of the soft actuator, it needs to be reinforced with fiber. The fiber is wrapped around the inner layer in such a way to perform the desired bending. For this paper, the wrapping was attached circularly with $45^{\circ}$ degrees angle. Then the role of the outer layer comes to the play. It was designed to be thicker than the inner layer with the same length. It has a length of $170.2 \mathrm{~mm}$ and diameter of $18 \mathrm{~mm}$. The $18 \mathrm{~mm}$ diameter contributes a radius of $9 \mathrm{~mm}$ which is $2 \mathrm{~mm}$ thicker than the inner layer. As a result, the thickness of the actuator is $4 \mathrm{~mm}$. This thickness is a sum of the inner layer thickness and the outer layer thickness.

\subsection{Mold Fabrication}

A 3D computer numerical control (CNC) milling machine was the tool used for printing the mold designs on a polyacetal material. This machine is called Roland MDX40A. The machine is connected to computer via USB cable. The software that is being used to interface between the computer and the $\mathrm{CNC}$ machine is called. Dr. Engrave.

The thickness of the polyacetal material is $12 \mathrm{~mm}$ whereas the chamber radius, as aforementioned, is $5 \mathrm{~mm}$. To print out the chamber mold on this material a strategy was taken into consideration. To obtain a $5 \mathrm{~mm}$ thickness rectangle on the surface of the material, we must let the machine drill down $7 \mathrm{~mm}$ on that specific rectangle. Figure 9 shows illustrates how the printing process was performed

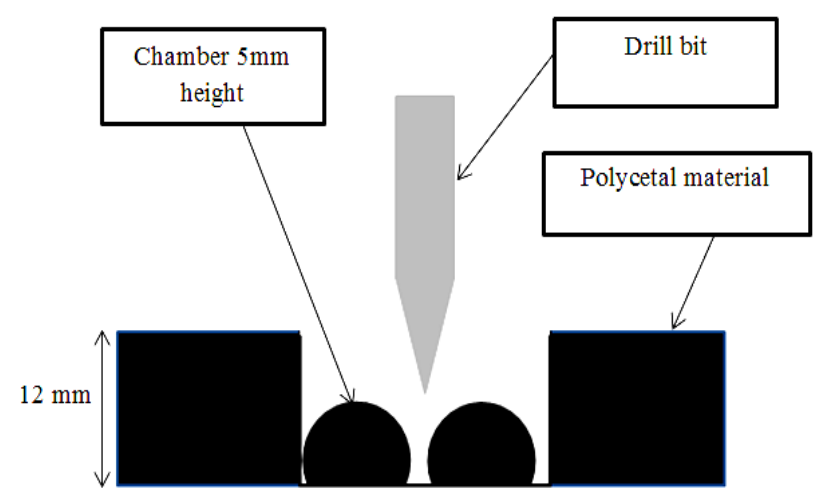

Figure 9. Strategy of printing the chambers

For other molds, such as the inner layer (refer to Figure 10 ), the joints (refer to Figure 11), the outer layer (refer to Figure 12), and the connector and the base molds (refer to Figure 13). The printing process was straightly done on that polyacetal material without the need to drill into a certain level of thickness. 

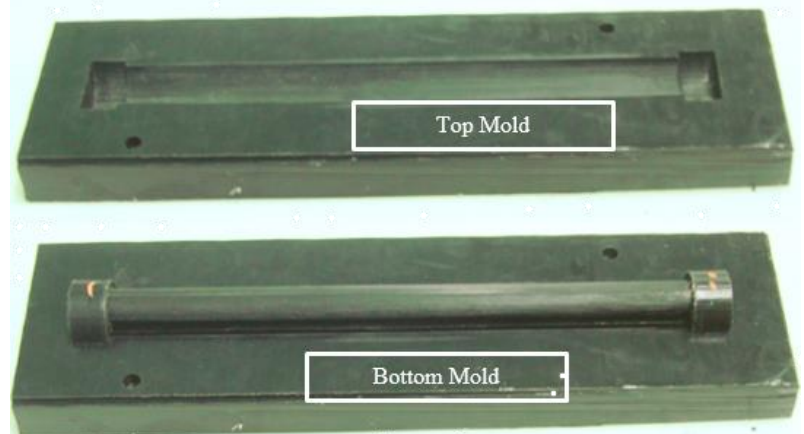

Figure 10. The inner layer mold

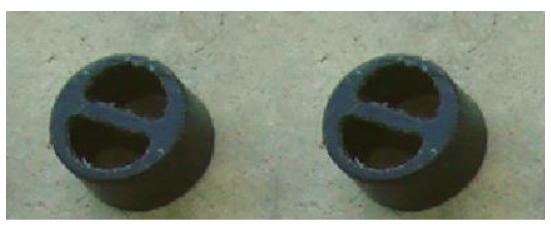

Figure 11. The joints mold

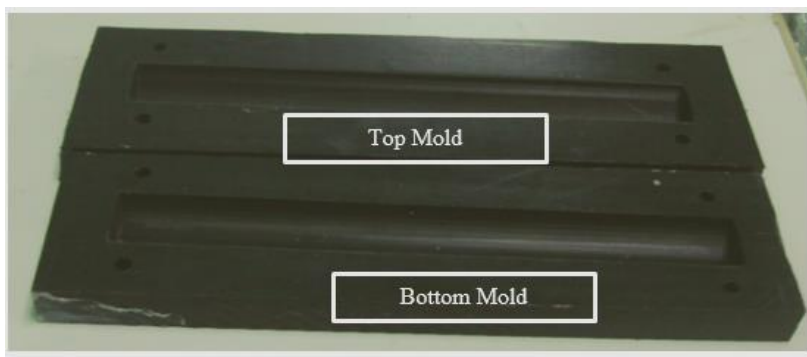

Figure 12. The outer layer mold
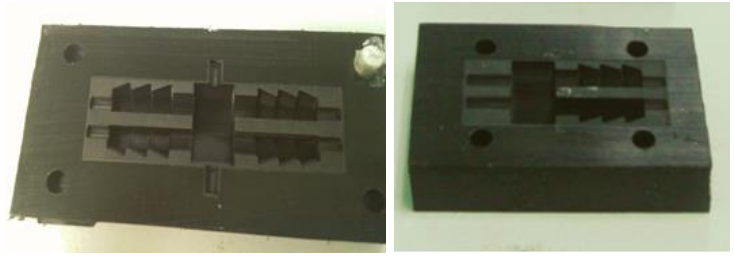

Figure 13. The connector and the base molds

\subsection{EXPERIMENTAL SETUP AND DISCUSSION}

The overall system is presented as in Figure 14. As there are six chambers, six solenoid valves are used to supply these chambers. An electrical circuit is presented to control the supplying valves. The black box shown in the figure consists a total of six switches. Each switch is connected to a valve. Turning on a switch will let the air flow into the chamber that is connected to the turned-on valve. Bending the manipulator in a certain direction requires turning on the desired valve to supply the desired chamber and yet result in the desired bending. A 24V DC supply is required to supply the valves. There is only one air pressure supply that is connected to common channel which relates all the six valves. The air pressure goes through a regulator before it is being fed to the common channel. The purpose of the regulator is to control the amount of pressure supplied by the air compressor.

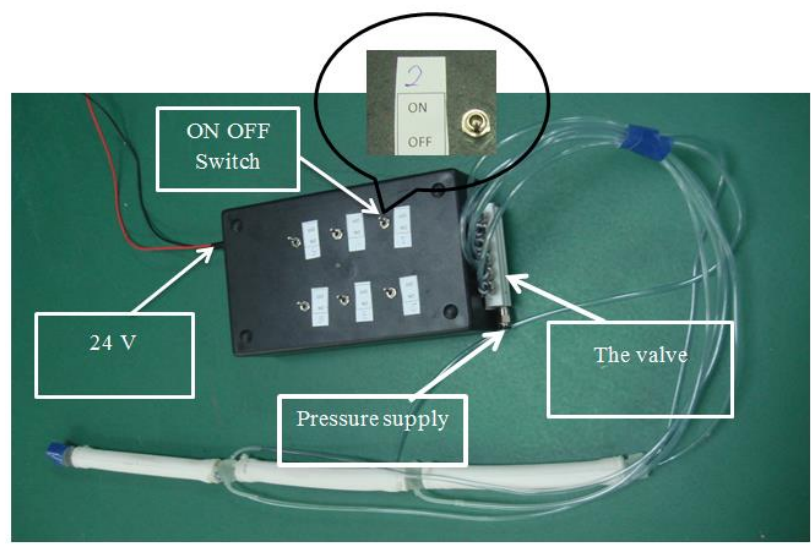

Figure 14. Overall manipulator along with the controller unit

\subsection{Single actuator bending test}

The manipulator was tested with different pressure values and in different directions. As a result, 8 possible movements can be performed by applying pressure in different possible chambers. Figure 15 shows the bending of one actuator when applying $150 \mathrm{kPa}$ at one of its chamber. Surprisingly, the actuators could take more pressure which resulted in a better bending. This can be also seen in Figure 15 where an input pressure of $170 \mathrm{kPa}$ and $180 \mathrm{kPa}$ were applied.

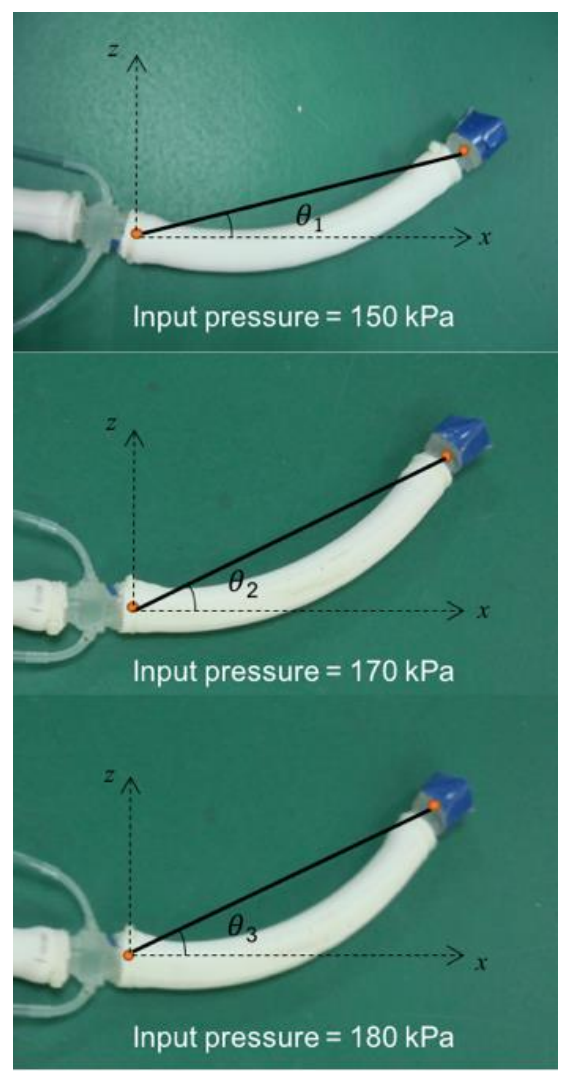

Figure 15. the bending angles due to input pressure of $150 \mathrm{kPa}, 170 \mathrm{kPa}$ and $180 \mathrm{kPa}$ 
Table 2. Comparison between experimental and simulated bending angle

\begin{tabular}{|c|c|c|}
\hline Pressure applied & Experimental $\theta$ & Simulated $\theta$ \\
\hline $150 \mathrm{kPa}$ & $15.07^{\circ}$ & $27.82^{\circ}$ \\
\hline $170 \mathrm{kPa}$ & $24.77^{\circ}$ & - \\
\hline $180 \mathrm{kPa}$ & $27.47^{\circ}$ & - \\
\hline
\end{tabular}

Table 2 above shows a comparison in bending angle between the experimental results and the simulation results. Bending angle calculation is shown in equation 4

$$
\theta_{1,2,3}=\tan ^{-1}\left(\begin{array}{l}
z \\
x
\end{array}\right)
$$

According to simulation, the maximum input pressure that can be pressurized into the chamber is $150 \mathrm{kPa}$ which results in a bending angle of $27.82^{\circ}$. When testing the same amount of pressure on the real chamber, the bending angle was $15.07^{\circ}$. This difference in the bending angle between the simulation and the experiment led to a curiosity whether the chamber can take more pressure or not. An input pressure of $170 \mathrm{kPa}$ was applied which resulted in a bending angle of $24.77^{\circ}$. At the simulation stage, the chamber failed to take this amount of pressure. The same thing is found when the pressure was raised to $180 \mathrm{kPa}$, the real actuator can take it and results in a better bending while the simulation fails to take this amount of pressure.

\subsection{Snake-like motion test}

The experiment was taken further to test the overall design of the manipulator. Figure 16 below shows the simulation result for applying $150 \mathrm{kPa}$ pressure in three chambers along the three links. The air was applied in chamber number $1,4,5$.

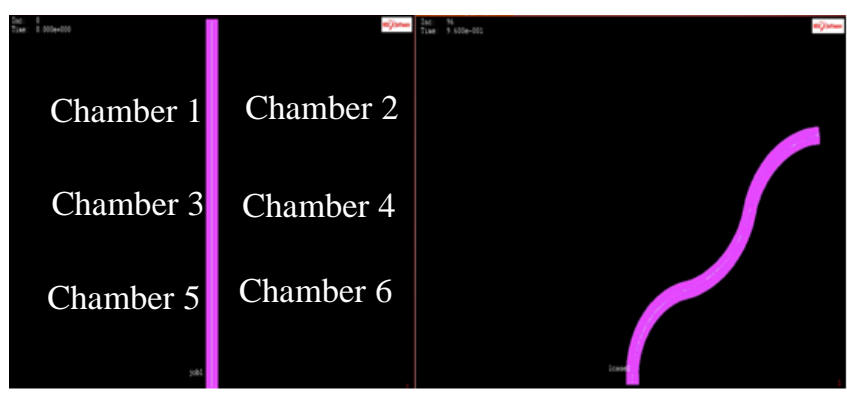

Figure 16. FEA of three actuator links

The same pressure was applied to the real manipulator to see whether it meets the simulation result. Figure 17 shows the bending of the manipulator when a pressure of $150 \mathrm{kPa}$ was applied to chambers the same order as it was applied in the simulation.

By comparing the above two figures, the bending is similar except the fact that the simulation bending is more obvious.

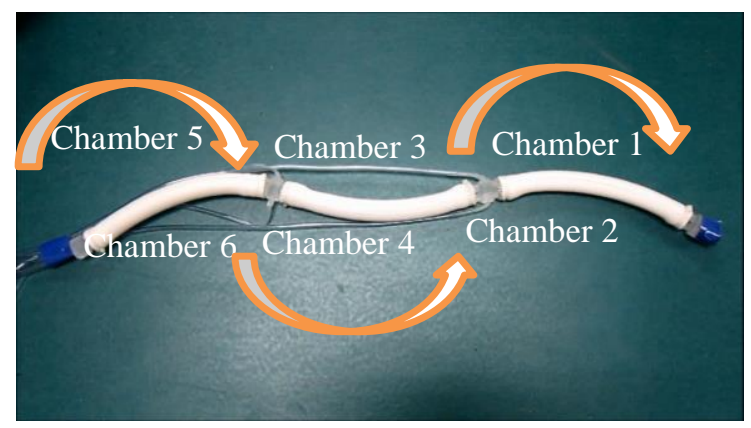

Figure 17: Different bending angle

\subsection{CONCLUSION}

The aim of this paper is to design and fabricate three links flexible rubber actuator. Results show that the fabricated flexible actuator can bend at higher air pressurization than the maximum pressure applied in the simulation software. The simulated flexible actuator stops running when more than $150 \mathrm{kPa}$ is applied whereas the fabricated actuator can perform bending motion until $180 \mathrm{kPa}$. One of the future improvements that can be done is by attaching a camera to the head of the snake to do image processing. With camera attachment to the three-links flexible manipulator design, it can be used as surveillance and checking robot. Another improvement is to link the manipulator with a real robot to act like an end effector. If the manipulator has a good flexibility, it can be used to hold objects. Another recommendation is by designing flexible manipulator from three internal chambers actuators to increase the degrees of freedom.

\section{REFERENCES}

[1] I. N. A. M. Nordin, A. A. Mohd Faudzi, M. Z Kamarudin, D. E. O. Dewi, T. Rehman, M. R. M. Razif, and AControl, "Grip Force Measurement of Soft-Actuated Finger Exoskeleton," vol. 78, no. 613, pp. 25-30, 2016.

[2] T. Ranzani, G. Gerboni, M. Cianchetti, and A. Menciassi, "A bioinspired soft manipulator for minimally invasive surgery.," Bioinspir. Biomim., vol. 10, no. 3, p. 35008, 2015.

[3] A. A. Mohd Faudzi, K. Suzumori, K. Satoshi, and S. Kurumaya, "Giacometti Six-legged Walking Robot - Leg Design Mechanism using Soft actuator -," Proc. 2016 JSME Conf. Robot. Mechatronics, p. 1A1-06b6(1-2), 2016.

[4] R. Niiyama, X. Sun, C. Sung, B. An, D. Rus, and K. Sangbae, "Pouch Motors : Printable Soft Actuators Integrated," Soft Robot., vol. 2, no. 2, pp. 59-71, 2015.

[5] T. Fukuda and M. Engneering, "Rubber Gas Actuator Driven by Hydrogen Storage Alloy for Inpipe Inspection Mobile Robot with Flexible Structure," pp. 1-6, 1989.

[6] A. A. Mohd Fauzi, K. Suzumori, and K. Satoshi, "Snake-Like Robot with Continuously Deformable Body Driven by Thin McKibben Muscles," Proc. 2016 JSME Conf. Robot. Mechatronics, p. 2PI12b1(1-2), 2016. 
[7] P. Liljebäck, Ø. Stavdahl, and A. Beitnes, "SnakeFighter - Development of a water hydraulic fire fighting snake robot," in 9th International Conference on Control, Automation, Robotics and Vision, 2006, ICARCV '06, 2006.

[8] Y. Wang, Z., Ma, S., Li, B. \& Wang, "Dynamic Modeling for Locomotion-Manipulation Of A Snake-Like Robot By Using Geometric Methods," pp. 3631-3636, 2009.

[9] M. Saito, M. Fukaya, and T. Iwasaki, "Serpentine Locomotion with Robotic Snakes," IEEE Control Syst., vol. 22, no. 1, pp. 64-81, 2002.

[10] J. Ostrowski and B. Joel, "Gait kinematics for a serpentine robot," Proc. IEEE Int. Conf. Robot. Autom., vol. 2, pp. 1294-1299, 1996.

[11] S. Ma, "Analysis of creeping locomotion of a snakelike robot," Adv. Robot., vol. 15, pp. 205-224, 2001.

[12] A. B. Andersson, "Discretization of a continuous curve," IEEE Trans. Robot., vol. 24, no. 2, pp. 456461, 2008.

[13] S. Wakimoto, J. Nakajima, M. Takata, T. Kanda, and K. Suzumori, "A micro snake-like robot for small pipe inspection," MHS 2003 - Proc. 2003 Int. Symp. Micromechatronics Hum. Sci., pp. 303-308, 2003.

[14] S. Coemert, A. Gao, J. P. Carey, M. F. Traeger, R. H. Taylor, T. C. Lueth, and M. Armand, "Development of a snake-like dexterous manipulator for skull base surgery," Proc. Annu. Int. Conf. IEEE Eng. Med. Biol. Soc. EMBS, vol. 2016-October, pp. 50875090, 2016.

[15] S. Koichi, L. Shoichi, and T. Hiroshisa, "Applying a Flexible Microactuator to Robotic Mechanisms," IEEE Control Syst., vol. 12, no. 1, pp. 21-27, 1992.

[16] M. De Volder, A. J. M. Moers, and D. Reynaerts, "Fabrication and control of miniature McKibben actuators," Sensors Actuators, A Phys., vol. 166, no. 1, pp. 111-116, 2011.

[17] T. Rehman, A. A. Mohd Faudzi, D. E. O. Dewi, K. Suzumori, M. R. M. Razif, and I. N. A. M. Nordin, "Design and Analysis of Bending Motion in Single and Dual Chamber Bellows," J. Teknol., vol. 78, no. 6-13, pp. 17-23, 2016.

[18] K. Kure, T. Kanda, K. Suzumori, and S. Wakimoto, "Flexible displacement sensor using injected conductive paste," Sensors Actuators, A Phys., vol. 143, no. 2, pp. 272-278, 2008.

[19] K. Suzumori, S. Iikura, and H. Tanaka, "Development of flexible microactuator and its applications to robotic mechanisms," in Proceedings. 1991 IEEE International Conference on Robotics and Automation, 1991, pp. 1622-1627.

[20] K. Suzumori, S. Iikura, H. Tanaka, T. Corporation, K. Suzumori, S. Iikura, and H. Tanaka, "Flexible microactuator for miniature robots," Micro Electro Mech. Syst. 1991, MEMS '91, Proceedings. An Investig. Micro Struct. Sensors, Actuators, Mach. Robot. IEEE, pp. 204-209, 1991. 